\title{
HELLY'S THEOREM AND SHIFTS OF SETS. II. SUPPORT FUNCTION, EXPONENTIAL SYSTEMS, ENTIRE FUNCTIONS
}

\author{
B.N. KHABIBULLIN
}

\begin{abstract}
Let $\mathcal{S}$ be a family of sets in $\mathbb{R}^{n}, S$ be the union of all these sets and $C$ be a convex set in $\mathbb{R}^{n}$. In terms of support functions of sets in $\mathcal{S}$ and set $C$ we establish necessary and sufficient conditions under which a parallel shift of set $C$ covers set $S$. We study independently the two-dimensional case, when sets are unbounded, by employing additional characteristics of sets. We give applications of these results to the problems of incompleteness of exponential systems in function spaces.
\end{abstract}

Keywords: convex set, system of linear inequalities, shift, support function, incompleteness of exponential systems, indicator of entire function

Mathematics Subject Classification: 52A35, 52A20

\section{INTRODUCTION AND SOME KEY RESULTS}

We use the notations of first part of work [1] and quite often, not mentioning separately, we employ known facts and notations from [2]-[6]. Nevertheless, in Section 1.1, for the sake of convenience, we recall elementary properties of support functions. Given $S \subset \mathbb{R}^{n}$, by cl $S$, int $S$, co $S$ we denote respectively closure, interior, convex hull/ of set $S ; B(x, r)$ stands for the open ball of radius $r>0$ in $\mathbb{R}^{n}$ centered at $x$.

1.1. For an arbitrary set $S \subset \mathbb{R}^{n}$,

$$
H_{S}: \mathbb{R}^{n} \rightarrow[-\infty,+\infty], \quad H_{S}(a):=\sup _{s \in S}\langle a, s\rangle, \quad a \in \mathbb{R}^{n},
$$

indicates the support function of set $S \subset \mathbb{R}^{n}$, where $\langle\cdot, \cdot\rangle$ denotes the scalar product in $\mathbb{R}^{n}$. In particular, if $S=\varnothing$ is the empty set in $\mathbb{R}^{n}$, then $H_{\varnothing}(a) \equiv-\infty, a \in \mathbb{R}^{n}$, and in accordance with usual convention $\sup \varnothing=-\infty$ and inf $\varnothing=+\infty$ for the empty subset in $[-\infty,+\infty]$. Vice versa, if $H_{S}(a)=-\infty$ for at least one $a \in \mathbb{R}^{n}$, then $S=\varnothing$. Thus, if $S \neq \varnothing$, then is the image $H_{S}\left(\mathbb{R}^{n}\right) \subset(-\infty,+\infty]$. Finally, set $S \subset \mathbb{R}^{n}$ is bounded if and only if $H_{S}\left(\mathbb{R}^{n}\right) \subset \mathbb{R}$.

Support function is positively homogeneous, i.e.,

$$
H_{S}(\lambda a) \equiv \lambda H_{S}(a), \quad \lambda \in(0,+\infty), \quad a \in \mathbb{R}^{n}, \quad \lambda \cdot( \pm \infty):= \pm \infty,
$$

is sub-additive, i.e., $H_{S}\left(a+a^{\prime}\right) \leqslant H_{S}(a)+H_{S}\left(a^{\prime}\right)$ for each $a, a^{\prime} \in \mathbb{R}^{n}$, is lower-semicontinuous and is even continuous, if $S$ is bounded and possesses topological-algebraic properties $H_{S}=$ $H_{\mathrm{cl} S}=H_{\mathrm{co} S}=H_{\mathrm{clco} S}, S \subset \mathbb{R}^{n}$, which can be complemented by the identities $H_{\operatorname{int} S}=H_{\operatorname{int} \operatorname{cl} S}=$ $H_{S}=H_{\text {clint } S}$ for convex $S$ as int $S \neq \varnothing$. It is clear that for a one-point set $S=\{x\}, x \in \mathbb{R}^{n}$ for each $a \in \mathbb{R}^{n}$ we have $H_{\{x\}}(a)=\langle x, a\rangle=\langle a, x\rangle$.

B.N. Khabibullin, Helly's Theorem and shifts of Sets. II. Support function, exponential SYSTEMS, ENTIRE FUNCTIONS.

(c) Khabibullin B.N. 2014.

The work is supported by RFBR (grant no. 13-01-00030-a).

Submitted February 25, 2014. 
For a convex set $C \subset \mathbb{R}^{n}$, set $S \subset \mathbb{R}^{n}$ is contained in $C$ for a closed $C$ or open $S$, if and only if $H_{S}(a) \leqslant H_{C}(a)$ for each $a \in \mathbb{R}^{n}$.

A set $S \subset C \subset \mathbb{R}^{n}$ is precompactly embedded into an open set $C$ if and only if the closure cl $S$ in the sense of the topology on $C$ inherited from $\mathbb{R}^{n}$ is a compact set in $C$ (we write $S \Subset C$ ).

1.2. The main studied problem for $S \subset \mathbb{R}^{n}$ and a convex $C \subset \mathbb{R}^{n}$ is to provide necessary and sufficient conditions ensuring that some shift of $S$ is contained in $C$, when $S$ is a union of arbitrary sets. At that, these conditions are to be given first of all in terms of support functions or in some functional way. The basis of our study is the following elementary

Proposition 1. Let $C$ be a non-empty convex set in $\mathbb{R}^{n}, S \subset \mathbb{R}^{n}$.

If $C$ is closed or $S$ is open, then some shift of set $S$ is contained in $C$ if and only if there exists $x \in \mathbb{R}^{n}$ such that $\langle a, x\rangle+H_{S}(a) \leqslant H_{C}(a)$ for each $a \in \mathbb{R}^{n}$.

For open $C$ some shift of $S$ is precompactly embedded into $C$ if and only if $S$ is bounded, i.e., $H_{S}\left(\mathbb{R}^{n}\right) \subset \mathbb{R}$ and there exists $x \in \mathbb{R}^{n}$ such that $\langle a, x\rangle+H_{S}(a)<H_{C}(a)$ for each $a \in \mathbb{R}^{n}$.

Proof. Some shift of $S$ is contained in $C$ if and only if there exists $x \in \mathbb{R}^{n}$ such that $S+x \subset C$. It yields $\langle a, x\rangle+H_{S}(a)=H_{S+x}(a) \leqslant H_{C}(a)$ for each $a \in \mathbb{R}^{n}$. And vice versa, if $C$ is closed or $S$ is open, then, as it was mentioned above, inclusion $S+x \subset C$ means $H_{S+x}(a) \leqslant H_{C}(a)$, where the left hand side if $\langle a, x\rangle+H_{S}(a)$. It completes the proof of the first part of the Proposition.

If $S$ is bounded, then $H_{S}$ is a continuous function and at that, $\langle\cdot, x\rangle+H_{S}-H_{C}$ is upper semicontinuous function and it attains its maximum $-\varepsilon<0$ on the unit sphere centered at 0 . By the positive homogeneity of the support function it implies $\langle a, x\rangle+H_{\mathrm{cl} S}(a)+\varepsilon|a| \leqslant H_{C}(a)$ for each $a \in \mathbb{R}^{n}$. Therefore, we have the inclusion $x+\operatorname{cl} S+\varepsilon B(0,1) \subset C$ and the compactness of $\operatorname{cl} S$ in $C$. The proof is complete.

1.3. In this subsection we provide some specific results for the case of a convex compact set $C \subset \mathbb{R}^{n}$ which is considered rather in details (see Theorems 1, 2 in the Introduction). Because of many possible subcases, the situation with an unbounded set $C \subset \mathbb{R}^{n}$ is considered only for some particular case (see Section 3, Subsection 3.1) and in more details for the planar case $n=2$, i.e., for $C \subset \mathbb{C}$, where the complex plane $\mathbb{C}$ is identified with $\mathbb{R}^{2}$ (see Section 3, Subsection 3.2). Cases of non-closed and non-open convex set $C$ are not touched at all as rather complicated even under the choice of an appropriate terminology. In Section 4 we prove theorems on incompleteness of exponential systems in various functional spaces demonstrating the importance of possibility of covering some union of sets by a shift of a convex set.

Theorem 1 (For convex sets $C \Subset \mathbb{R}^{n}$ ). Let $n \in \mathbb{N}, C$ be a convex bounded set in $\mathbb{R}^{n}$, $\mathscr{S}$ be the family of sets in $\mathbb{R}^{n}$, and $S$ be the union of all sets in $\mathscr{S}$. We assume that $C$ is closed or $S$ is open. Then the following four statements are mutually equivalent:

1. some shift of set $S$ is contained in $C$;

2. for each $n+1$ sets $S_{1}, \ldots, S_{n+1}$ in family $\mathscr{S}$ and each $n+1$ closed half-spaces $C_{1}, \ldots C_{n+1}$ containing $C$ and bounded by support hyperplanes of set $C$, there exists a vector $x$ such that each shift $S_{k}+x$ is contained in closed half-space $C_{k}$ for each $k=1, \ldots, n+1$;

3. for each $n+1$ sets $S_{1}, \ldots, S_{n+1}$ in family $\mathscr{S}$ and each $n+1$ vectors $a_{1}, \ldots, a_{n+1} \in \mathbb{R}^{n}$ and numbers $p_{1}, \ldots, p_{n+1} \geqslant 0$, the identity

$$
\sum_{k=1}^{n+1} p_{k} H_{S_{k}}\left(a_{k}\right) \leqslant \sum_{k=1}^{n+1} p_{k} H_{C}\left(a_{k}\right)
$$

holds true under condition

$$
\sum_{k=1}^{n+1} p_{k} a_{k}=0
$$


4. for each $n+1$ sets $S_{1}, \ldots, S_{n+1}$ in family $\mathscr{S}$ and each $n+1$ vectors

$$
\left\{\begin{array}{l}
a_{1}=\left(a_{11}, \ldots, a_{1 n}\right) \in \mathbb{R}^{n} \\
\left.\ldots \ldots \ldots \ldots \ldots, \ldots \ldots, a_{n+1, n}\right) \in \mathbb{R}^{n} \\
a_{n+1}=\left(a_{n+1,1}, \ldots \ldots\right.
\end{array}\right.
$$

of rank $r>0$ there exists a non-zero minor

$$
\Delta=\left|\begin{array}{ccc}
a_{k_{1} j_{1}} & \cdots & a_{k_{1} j_{r}} \\
\vdots & & \vdots \\
a_{k_{r} j_{1}} & \cdots & a_{k_{r} j_{r}}
\end{array}\right|
$$

or $r$ th order satisfying inequalities $(k=1, \ldots, n+1)$

$$
\frac{1}{\Delta}\left|\begin{array}{cccc}
a_{k_{1} j_{1}} & \cdots & a_{k_{1} j_{r}} & H_{S_{k_{1}}}\left(a_{k_{1}}\right) \\
\vdots & & \vdots & \vdots \\
a_{k_{r} j_{1}} & \cdots & a_{k_{r} j_{r}} & H_{S_{k_{r}}}\left(a_{k_{r}}\right) \\
a_{k j_{1}} & \cdots & a_{k j_{r}} & H_{S_{k}}\left(a_{k}\right)
\end{array}\right| \leqslant \frac{1}{\Delta}\left|\begin{array}{cccc}
a_{k_{1} j_{1}} & \cdots & a_{k_{1} j_{r}} & H_{C}\left(a_{k_{1}}\right) \\
\vdots & & \vdots & \vdots \\
a_{k_{r} j_{1}} & \cdots & a_{k_{r} j_{r}} & H_{C}\left(a_{k_{r}}\right) \\
a_{k_{1}} & \cdots & a_{k j_{r}} & H_{C}\left(a_{k}\right)
\end{array}\right| .
$$

In the planar case $n=2$ we identify $\mathbb{R}^{2}$ with the complex plane $\mathbb{C}$ :

$$
\mathbb{R}^{2} \ni(x, y) \mapsto x+i y=: z:=r e^{i \theta} \in \mathbb{C}, \quad i \text { is the imaginary unit, } r \geqslant 0, \theta \in \mathbb{R} .
$$

At that, it is a tradition [7, 8] that instead of support $H_{S}$ one considers a $2 \pi$-periodic function $h_{S}: \mathbb{R} \rightarrow[-\infty,+\infty]$ called also support function for $S \subset \mathbb{C}$ and defined by the restriction of $H_{S}$ on the unit circle

$$
h_{S}(\theta):=H_{S}\left(e^{i \theta}\right)=\sup _{s \in S} \operatorname{Re} s e^{-i \theta}, \quad \theta \in \mathbb{R} .
$$

In view of positive homogeneity (1) of function $H_{S}$, it is uniquely recovered by function $h_{S}$, and Theorem 1 casts into the form:

Theorem 2 (for convex sets $C \Subset \mathbb{C}$ ). Let $C$ be a convex bounded set in $\mathbb{C}, \mathscr{S}$ is a family of sets in $\mathbb{C}$, and $S$ is the union of all sets in $\mathscr{S}$. Suppose that $C$ is closed or $S$ is open. Then the following four statements are mutually equivalent:

1. some shift of set $S$ is contained in $C$;

2. for each three sets $S_{1}, S_{2}, S_{3}$ in $\mathscr{S}$ and each closed non-empty triangl 1 enveloping $C$, there exists a point $z \in \mathbb{C}$ such that all three shifts $S_{1}+z, S_{2}+z, S_{3}+z$ are contained in this triangle;

3. for each three sets $S_{1}, S_{2}, S_{3} \in \mathscr{S}$ and each three numbers $\theta_{1}, \theta_{2}, \theta_{3} \in \mathbb{R}$ and numbers $q_{1}, q_{2}, q_{3} \geqslant 0$ the inequality

$$
q_{1} h_{S_{1}}\left(\theta_{1}\right)+q_{2} h_{S_{2}}\left(\theta_{2}\right)+q_{3} h_{S_{3}}\left(\theta_{3}\right) \leqslant q_{1} h_{C}\left(\theta_{1}\right)+q_{2} h_{C}\left(\theta_{2}\right)+q_{3} h_{C}\left(\theta_{3}\right)
$$

holds true under the condition

$$
q_{1} e^{i \theta_{1}}+q_{2} e^{i \theta_{2}}+q_{3} e^{i \theta_{3}}=0
$$

4. for each three sets $S_{1}, S_{2}, S_{3} \in \mathscr{S}$ and each numbers $\theta_{1}, \theta_{2}, \theta_{3} \in \mathbb{R}$ both the conditions hold: (a) if each difference of the above numbers is a multiple of $\pi$, then for each pair $k, j \in$ $\{1,2,3\}$ whose difference $\theta_{j}-\theta_{k}$ is not a multiple of $2 \pi$, the inequality

$$
h_{S_{1}}\left(\theta_{k}\right)+h_{S_{2}}\left(\theta_{j}\right) \leqslant h_{C}\left(\theta_{k}\right)+h_{C}\left(\theta_{j}\right)
$$

holds true;

\footnotetext{
${ }^{1}$ As a triangle we also treat a non-degenerate segment (just one side of zero length), a point (all sides have zero length and all the vertices coincide), and a figure bounded by parallel lines and an intersecting them line (one vertex is point $\infty$, two sides are of infinite length).
} 
(b) if, possibly after renumbering, the difference $\theta_{2}-\theta_{1}$ is not a multiple of $\pi$, then the inequality

$$
\begin{aligned}
h_{S_{1}}\left(\theta_{1}\right) \frac{\sin \left(\theta_{3}-\theta_{2}\right)}{\sin \left(\theta_{2}-\theta_{1}\right)}+h_{S_{3}}\left(\theta_{3}\right) & +h_{S_{2}}\left(\theta_{2}\right) \frac{\sin \left(\theta_{1}-\theta_{3}\right)}{\sin \left(\theta_{2}-\theta_{1}\right)} \\
& \leqslant h_{C}\left(\theta_{1}\right) \frac{\sin \left(\theta_{3}-\theta_{2}\right)}{\sin \left(\theta_{2}-\theta_{1}\right)}+h_{C}\left(\theta_{3}\right)+h_{C}\left(\theta_{2}\right) \frac{\sin \left(\theta_{1}-\theta_{3}\right)}{\sin \left(\theta_{2}-\theta_{1}\right)} .
\end{aligned}
$$

holds true.

\section{Proofs of Theorems 1 and 2}

Proof of Theorem 1. The implication $1 \Rightarrow 2$ is obvious.

In order to prove the implication $2 \Rightarrow 1$, for each vector $a \in \mathbb{R}^{n}, a \neq 0$, by $C_{a}$ we denote the closed half-space containing $C$ and bounded by the support hyperplane to convex set $C$ in the direction of $a$, i.e.,

$$
C_{a}:=\left\{x:\langle x, a\rangle \leqslant H_{C}(a)\right\} .
$$

Here $C_{a}=C_{a^{\prime}}$ if vectors $a$ and $a^{\prime}$ are codirectional, i.e., $a=\alpha a^{\prime}$ for some $\alpha>0$. We consider the family of half-spaces $\mathscr{C}:=\left\{C_{a}: a \in \mathbb{R}^{n} \backslash\{0\}\right\}$, where the intersection $C=\bigcap_{a \neq 0} C_{a}$ is bounded. Statement 2 of the theorem means that for each $n+1$ sets $S_{1}, \ldots, S_{n+1}$ in family $\mathscr{S}$ and each $n+1$ closed subspaces $C_{a_{1}}, \ldots C_{a_{n+1}}$ there exists a vector $x$ such that each shift $S_{k}+x$ is contained in closed half-space $C_{a_{k}}$ for each $k=1, \ldots, n+1$, i.e., the intersection

$$
\bigcap_{k=1}^{n+1}\left(S_{k} * C_{a_{k}}\right)
$$

of the geometric differences $S_{k} * C_{a_{k}}$ is non-empty. Then 1, Thm. 1, Rem. 2, Implication $(\mathrm{CS}) \Rightarrow(\mathrm{T})$ ] implies the implication $2 \Rightarrow 1$ of the theorem.

In order to prove the equivalence $2 \Leftrightarrow 3$, we rewrite statement 2 as a system of $n+1$ linear inequalities. By Proposition 1, Statement 2 is equivalent to an infinite series of inequalities

$$
\langle a, x\rangle+H_{S_{k}}(a) \leqslant H_{C_{a_{k}}}(a) \text { for each } a \in \mathbb{R}^{n}, k=1, \ldots, n+1 .
$$

But in accordance with the definition of closed subspaces $C_{a_{k}}$, for each vector $a$ not codirectional with $a_{k}$, we have $H_{C_{a_{k}}}(a)=+\infty$. Therefore, infinite system of inequalities (8) is equivalent to the finite system $n+1$ of linear inequalities

$$
\left\langle a_{k}, x\right\rangle+H_{S_{k}}\left(a_{k}\right) \leqslant H_{C_{a_{k}}}\left(a_{k}\right) \text { for each } a_{k} \in \mathbb{R}^{n}, k=1, \ldots, n+1,
$$

or in the traditional notation

$$
\left\langle a_{k}, x\right\rangle-\left(H_{C_{a_{k}}}\left(a_{k}\right)-H_{S_{k}}\left(a_{k}\right)\right) \leqslant 0 \quad \text { for each } a_{k} \in \mathbb{R}^{n}, k=1, \ldots, n+1 .
$$

By the known Aleksandrov-Fan-Chi theorem [9, Thm. 2.3] is equivalent to the statement: for each $n+1$ numbers $p_{1}, \ldots, p_{n+1} \geqslant 0$, under the condition

$$
\sum_{k=1}^{n+1} p_{k} a_{k}=0,
$$

the inequality

$$
\sum_{k=1}^{n+1} p_{k}\left(H_{S_{k}}\left(a_{k}\right)-H_{C}\left(a_{k}\right)\right) \leqslant 0
$$

holds true. The latter is equivalent to statement 3 of the theorem.

Returning back to finite system of $n+1$ linear inequalities (9) (for each fixed set of vectors $a_{1}, \ldots, a_{n+1} \in \mathbb{R}^{n}$ ) by S.N. Chernikov solvability criterion [9, Thm. 1.5] for finite system of 
linear inequalities, in notations and conventions (2)-(3), system (9) is solvable if and only if the inequality

$$
\left|\begin{array}{cccc}
a_{k_{1} j_{1}} & \cdots & a_{k_{1} j_{r}} & H_{C}\left(a_{k_{1}}\right)-H_{S_{k_{1}}}\left(a_{k_{1}}\right) \\
\vdots & & \vdots & \vdots \\
a_{k_{r} j_{1}} & \cdots & a_{k_{r} j_{r}} & H_{C}\left(a_{k_{r}}\right)-H_{S_{k_{r}}}\left(a_{k_{r}}\right) \\
a_{k j_{1}} & \cdots & a_{k j_{r}} & H_{C}\left(a_{k}\right)-H_{S_{k}}\left(a_{k}\right)
\end{array}\right| \geqslant 0, \quad k=1, \ldots, n+1,
$$

holds true that coincides with inequality (4). The proof is complete.

Proof of Theorem 2. It is easy to see that statements 1 and 2 are exactly statements 1 and 2 of Theorem 1. In order to obtain statement 3 of Theorem 2, in view of (5) we write statement 3 of Theorem 1 for $n=2$ as follows: for each three sets $S_{1}, S_{2}, S_{3} \in \mathscr{S}$ and each three numbers $a_{1}=t_{1} e^{i \theta_{2}}, a_{2}=t_{2} e^{i \theta_{2}}, a_{3}=t_{3} e^{i \theta_{3}} \in \mathbb{C}$, where $t_{1}, t_{2}, t_{3}>0$, and $p_{1}, p_{2}, p_{3} \geqslant 0$, under the condition

the inequality

$$
p_{1} t_{1} e^{i \theta_{1}}+p_{2} t_{2} e^{i \theta_{2}}+p_{3} t_{3} e^{i \theta_{3}}=0
$$

$$
p_{1} t_{1} h_{S_{1}}\left(\theta_{1}\right)+p_{2} t_{2} h_{S_{2}}\left(\theta_{2}\right)+p_{3} t_{3} h_{S_{3}}\left(\theta_{3}\right) \leqslant p_{1} t_{1} h_{C}\left(\theta_{1}\right)+p_{2} t_{2} h_{C}\left(\theta_{2}\right)+p_{3} t_{3} h_{C}\left(\theta_{3}\right)
$$

holds true. Letting $q_{1}=p_{1} t_{1}, q_{2}=p_{2} t_{2}, q_{3}=p_{3} t_{3}$, by the positive homogeneity (1) we make sure that the latter statement is equivalent to statement 3 of Theorem 2 .

Let us prove that statement 4 of Theorem 1 as $n=2$ coincides with statement 4 of Theorem 2 .

We can treat three vectors $a_{1}, a_{2}, a_{3} \in \mathbb{R}^{2}$ in $(2)$ as three complex numbers

$$
\begin{cases}a_{1}:=t_{1} e^{i \theta_{1}}=t_{1} \cos \theta_{1}+i \cdot t_{1} \sin \theta_{1}, & t_{1}>0, \\ a_{2}:=t_{2} e^{i \theta_{2}}=t_{2} \cos \theta_{2}+i \cdot t_{2} \sin \theta_{2}, & t_{2}>0, \\ a_{3}:=t_{3} e^{i \theta_{3}}=t_{3} \cos \theta_{3}+i \cdot t_{3} \sin \theta_{3}, & t_{3}>0 .\end{cases}
$$

The rank is considered over field $\mathbb{R}$.

Case of rank $r=1$. In this case radius vectors of the point are codirectional or opposite directed. In the case when the radius vecotrs are codirectional, all the six differences $\theta_{j}-\theta_{k}$, $j, k=1,2,3, j \neq k$, are multiples of $2 \pi$, both sides of inequality (4) vanish by $2 \pi$-periodicity of function (5) and inequality (4) holds true immediately. Suppose that at least two vectors are opposite directed. For the sake of definiteness assume that these are $a_{1}$ and $a_{2}$, i.e., $\theta_{2}-\theta_{1}$ is a multiple of $\pi$ but not a multiple of $2 \pi$ and again for simplicity $\Delta=t_{1} \cos \theta_{1} \neq 0$. Then (4) is rewritten as

$$
\frac{1}{t_{1} \cos \theta_{1}}\left|\begin{array}{ll}
t_{1} \cos \theta_{1} & H_{S_{1}}\left(t_{1} e^{i \theta_{1}}\right) \\
t_{k} \cos \theta_{k} & H_{S_{k}}\left(t_{k} e^{i \theta_{k}}\right)
\end{array}\right| \leqslant \frac{1}{t_{1} \cos \theta_{1}}\left|\begin{array}{cc}
t_{1} \cos \theta_{1} & H_{C}\left(t_{1} e^{i \theta_{1}}\right. \\
t_{k} \cos \theta_{k} & H_{C}\left(t_{k} e^{i \theta_{k}}\right)
\end{array}\right|
$$

where $k=2,3$, or, in view of (5),

$$
\frac{1}{\cos \theta_{1}}\left|\begin{array}{cc}
\cos \theta_{1} & h_{S_{1}}\left(\theta_{1}\right) \\
\cos \theta_{k} & h_{S_{k}}\left(\theta_{k}\right)
\end{array}\right| \leqslant \frac{1}{\cos \theta_{1}}\left|\begin{array}{cc}
\cos \theta_{1} & h_{C}\left(\theta_{1}\right) \\
\cos \theta_{k} & h_{C}\left(\theta_{k}\right)
\end{array}\right| .
$$

If $\theta_{3}-\theta_{1}$ is a multiple of $2 \pi$, then both sides of the latter inequality vanish and it obviously holds. If the difference $\theta_{k}-\theta_{1}$ is a multiple of $\pi$ but not of $2 \pi$, then $\cos \theta_{k}=-\cos \theta_{1}$. Thus in this case it follows from (11) that

$$
h_{S_{k}}\left(\theta_{k}\right)-\frac{\cos \theta_{k}}{\cos \theta_{1}} h_{S_{1}}\left(\theta_{1}\right) \leqslant h_{C}\left(\theta_{k}\right)-\frac{\cos \theta_{k}}{\cos \theta_{1}} h_{S_{1}}\left(\theta_{1}\right) .
$$

It implies inequality (6) with $j=1$. Since $S_{1}, S_{2}, S_{3}$ are arbitrary, in the left hand side of (12) we can take each of the sets $S_{1}, S_{2}, S_{3}$ instead of $S_{k}$ and $S_{1}$. In the same way we proceed if $\cos \theta_{1}=0$, but we use $\sin \theta_{1} \neq 0$. The search of the other cases for $r=1$ is reduced to renumeration of numbers and sets. Hence, we arrive at item 4(a). 
Case of rank $r=2$. Suppose that two radius vectors of the points in $(10)$ are linearly independent, say, $a_{1}$ and $a_{2}$. It means that

$$
\Delta:=\left|\begin{array}{cc}
t_{1} \cos \theta_{1} & t_{2} \sin \theta_{2} \\
t_{1} \cos \theta_{1} & t_{2} \sin \theta_{2}
\end{array}\right|=t_{1} t_{2} \sin \left(\theta_{2}-\theta_{1}\right) \neq 0
$$

At that, inequality (4) is written as

$$
\begin{gathered}
\frac{1}{t_{1} t_{2} \sin \left(\theta_{2}-\theta_{1}\right)}\left|\begin{array}{ccc}
t_{1} \cos \theta_{1} & t_{1} \sin \theta_{1} & t_{1} h_{S_{1}}\left(\theta_{1}\right) \\
t_{2} \cos \theta_{2} & t_{2} \sin \theta_{2} & t_{2} h_{S_{2}}\left(\theta_{2}\right) \\
t_{3} \cos \theta_{3} & t_{3} \sin \theta_{3} & t_{3} h_{S_{3}}\left(\theta_{3}\right)
\end{array}\right| \\
\leqslant \frac{1}{t_{1} t_{2} \sin \left(\theta_{2}-\theta_{1}\right)}\left|\begin{array}{lll}
t_{1} \cos \theta_{1} & t_{1} \sin \theta_{1} & t_{1} h_{C}\left(\theta_{1}\right) \\
t_{2} \cos \theta_{2} & t_{2} \sin \theta_{2} & t_{2} h_{C}\left(\theta_{2}\right) \\
t_{3} \cos \theta_{3} & t_{3} \sin \theta_{3} & t_{3} h_{C}\left(\theta_{3}\right)
\end{array}\right|,
\end{gathered}
$$

or

$$
\frac{1}{\sin \left(\theta_{2}-\theta_{1}\right)}\left|\begin{array}{ccc}
\cos \theta_{1} & \sin \theta_{1} & h_{S_{1}}\left(\theta_{1}\right) \\
\cos \theta_{2} & \sin \theta_{2} & h_{S_{2}}\left(\theta_{2}\right) \\
\cos \theta_{3} & \sin \theta_{3} & h_{S_{3}}\left(\theta_{3}\right)
\end{array}\right| \leqslant \frac{1}{\sin \left(\theta_{2}-\theta_{1}\right)}\left|\begin{array}{lll}
\cos \theta_{1} & \sin \theta_{1} & h_{C}\left(\theta_{1}\right) \\
\cos \theta_{2} & \sin \theta_{2} & h_{C}\left(\theta_{2}\right) \\
\cos \theta_{3} & \sin \theta_{3} & h_{C}\left(\theta_{3}\right)
\end{array}\right|
$$

Expanding two latter determinants along the last columns, we obtain (7) that completes the proof of Theorem 2 .

\section{UnBounded CONVEX CLOSED SET $C$}

3.1. Case $n \geqslant 1$. In particular rather simple situations analogues of Theorems 1 and 2 can be established for an unbounded domain $C \subset \mathbb{R}^{n}$. We recall [1, Def. 1] that a non-zero vector $y \in \mathbb{R}^{n}$ a star-shapedness direction (w.r.t. the infinity), or is called a recession direction if for each point $c \in C$ the ray $r_{y}(c):=\{c+t y: t \geqslant 0\}$ is contained in $C$. A vector $y \in \mathbb{R}^{n}$ is called linearity direction if both $y$ and the opposite vector $-y$ are star-shapedness directions for set $C$, i.e., for each point $c \in C$ the straight line

$$
l_{y}(c):=\{c+t y: t \in \mathbb{R}\}=r_{y}(c) \cup\left(r_{-y}(c)\right)=l_{-y}(c)
$$

is contained in $C$. Set $C$ is polyhedral, if $C$ is the intersection of a finite number of closed half-spaces defined by a finite system of linear inequalities

$$
\langle a, x\rangle-b \leqslant 0 \quad \text { for some } a \in \mathbb{R}^{n}, \quad b \in \mathbb{R} .
$$

At that, the half-space defined by (13) are called determining half-spaces of polyhedral set $C$.

Theorem 3 (for unbounded convex closed sets). Let $C$ be a convex unbounded closed set in $\mathbb{R}^{n}, n \in \mathbb{N}, \mathscr{S}$ be a family of sets in $\mathbb{R}^{n}$, and $S$ be the union of all sets in $\mathscr{S}$. Suppose that family $\mathscr{S}$ is finite, i.e., card $\mathscr{S}<\infty$, and set $C$ is polyhedral or each star-shapedness direction for $C$ is the linearity direction for $C$. Then the following four statements are mutually equivalent:

1. some shift of set $S$ is contained in $C$;

2. for each $n+1$ sets $S_{1}, \ldots, S_{n+1}$ in family $\mathscr{S}$ and each $n+1$ closed half-spaces (only determining if $C$ is polyhedral ) $C_{1}, \ldots C_{n+1}$ containing $C$ and bounded by support hyperplanes to convex set $C$, there exists a vector $x$ such that each shift $S_{k}+x$ is contained in closed half-space $C_{k}$ for each $k=1, \ldots, n+1$;

3. statement 3 of Theorem 1 holds true;

4. statement $\overline{4}$ of Theorem $\overline{1}$ holds true.

Proof. Let $\mathscr{C}$ be a finite family of all determining half-spaces when $C$ is a polyhedral set, or, otherwise, be the family of all closed half-spaces containing $C$ and bounded by support hyperplanes to convex set $C$. Applying [1, Thm. 1 on covering by shifts, Cond. (F)], under 
the finiteness condition ( $C$ is a polyhedral set, family $\mathscr{S}$ is finite), the equivalence $(\mathrm{ST}) \Leftrightarrow(\mathrm{T})$ in [1, Thm. 1] implies the equivalence $1 \Leftrightarrow 2$. Under our assumptions for the star-shapedness conditions we employ [1, Thm. 1 on covering by shifts, Cond. (d) with card $\mathscr{S}<\infty$ ] and again the equivalence $(\mathrm{ST}) \Leftrightarrow(\mathrm{T})$ in $[1$, Thm. 1] yields the equivalence $1 \Leftrightarrow 2$, The rest of the proof (of the equivalence $2 \Leftrightarrow 3$ and $2 \Leftrightarrow 4$ of the theorem reproduces the proof of similar equivalence in Theorem 1 with no major changes.

3.2. Planar case. We recall that the breadth $B_{S}(\theta)$ (see [10, 33], [11, 4.1.1], [12, Ch. I, Sect. 4]) of an arbitrary set $S \subset \mathbb{C}$ in the direction of $\theta \in \mathbb{R}$ is the distance between two support lines to $S$ orthogonal to the radius vector $e^{i \theta}$. In terms of the support function we have

$$
B_{S}(\theta)=h_{S}(\theta)+h_{S}(\theta+\pi)=H_{S}\left(e^{i \theta}\right)+H_{S}\left(-e^{i \theta}\right) .
$$

The smallest breadth $b_{S}:=\inf _{\theta \in \mathbb{R}} B_{S}(\theta)$ is called width [12, Ch I, Sect. 4] or thickness (germ. 'dicke') [11, 4.1.1] of set $S$. If $e^{i \theta}$ is the star-shapedness direction for a convex set $C \subset \mathbb{C}$, then it is convenient to call number $\theta \in \mathbb{R}$ the star-shapedness direction as well. This is how we shall treat the star-shapedness direction in the planar case. Under such definition, number $\theta$ is a linearity direction if both $\theta$ and $\theta+\pi$ are star-shapedness directions. If each star-shapedness direction of a convex unbounded closed set $C \subset \mathbb{C}$ is a linearity direction, then it is either the empty set or whole complex plane or a strip of a finite width, i.e., in each case $C$ is a polyhedral set or a convex polygon in a general sense (respectively, either with no vertices and sides, or one-angle with the vertex at $\infty$ and the side of zero length, or two-angle with the vertices $\infty$ and two-sides of infinite length). Thus, in Theorem 3 condition for the star-shapedness directions of set $C$ is included in the case of its polyhedrality and as $n=2$, Theorem 3 becomes shorter:

Theorem 4 (for unbounded convex sets $C \subset \mathbb{C}$ ). Let $C$ be a convex unbounded closed polygon in $\mathbb{C}$ (in the general sense, with a finite amount of sides among those there can be sides of infinite length, i.e., rays or straight lines), $\mathscr{S}$ be a finite family of sets in $\mathbb{C}$, and $S$ be the union of all sets in family $\mathscr{S}$. Then the following four statements are mutually equivalent:

1. some shift of set $S$ is contained in $C$;

2. for all sets $S_{1}, S_{2}, S_{3}$ in family $\mathscr{S}$ and each closed triangle (in the general sense, with sides determining polyhedral set $C$ ) there exists a point $z \in \mathbb{C}$ such that shifts $S_{k}+z$, $k=1,2,3$, are contained in this triangle;

3. statement 3 of Theorem 2 holds true;

4. statement 4 of Theorem 2 holds true.

But there are a lot situations when instructive statements are possible in a simpler form or not for a polyhedral unbounded convex set $C$. Some of them were employed, sometimes implicitly, in works [13, [14, Sect. 7], [15, Sect. 4] (see also [16, Subsect. 3.2.1-3.2.3]) in studying of completeness of exponential systems in the spaces of functions on unbounded convex sets.

Given a convex set $C \subset \mathbb{C}$, its recession arc or star-shapedness arc (w.r.t. the infinity) is the arc of the unit circumference formed by intersection of this unit circumference with the set of all the star-shapedness directions for $C$ [2, Ch. II, Sect. 8]. The star-shapedness arc is denoted by $0^{+} C$. Set $C$ is bounded if and only if its star-shapedness arc is an empty set [2, Ch. II, Thm. 8.4]. If the star-shapedness arc of a convex set $C$ involves an arc of angle $>\pi$, then $C=\mathbb{C}$. For an arbitrary set $S \subset \mathbb{C}$ we also define the star-shapedness arc $0^{+} S:=0^{+} \operatorname{co} S$.

Let $S \subset \mathbb{C}$. We define functions of cut upper and lower width of set $S$ w.r.t. a point $s$ in the direction $\theta=0$ by the rule

$$
\begin{cases}W_{S}^{\uparrow}(x ; s):=\sup \{\operatorname{Im} z-\operatorname{Im} s: z \in S, \operatorname{Im} z \geqslant \operatorname{Im} s, \operatorname{Re} z=x\}, & x \in \mathbb{R}, \\ W_{S}^{\downarrow}(x ; s):=\sup \{\operatorname{Im} s-\operatorname{Im} z: z \in S, \operatorname{Im} z \leqslant \operatorname{Im} s, \operatorname{Re} z=x\}, & x \in \mathbb{R},\end{cases}
$$

where, as usually, $\sup \varnothing:=-\infty$ for the empty subset $\varnothing \subset[-\infty,+\infty]$. 


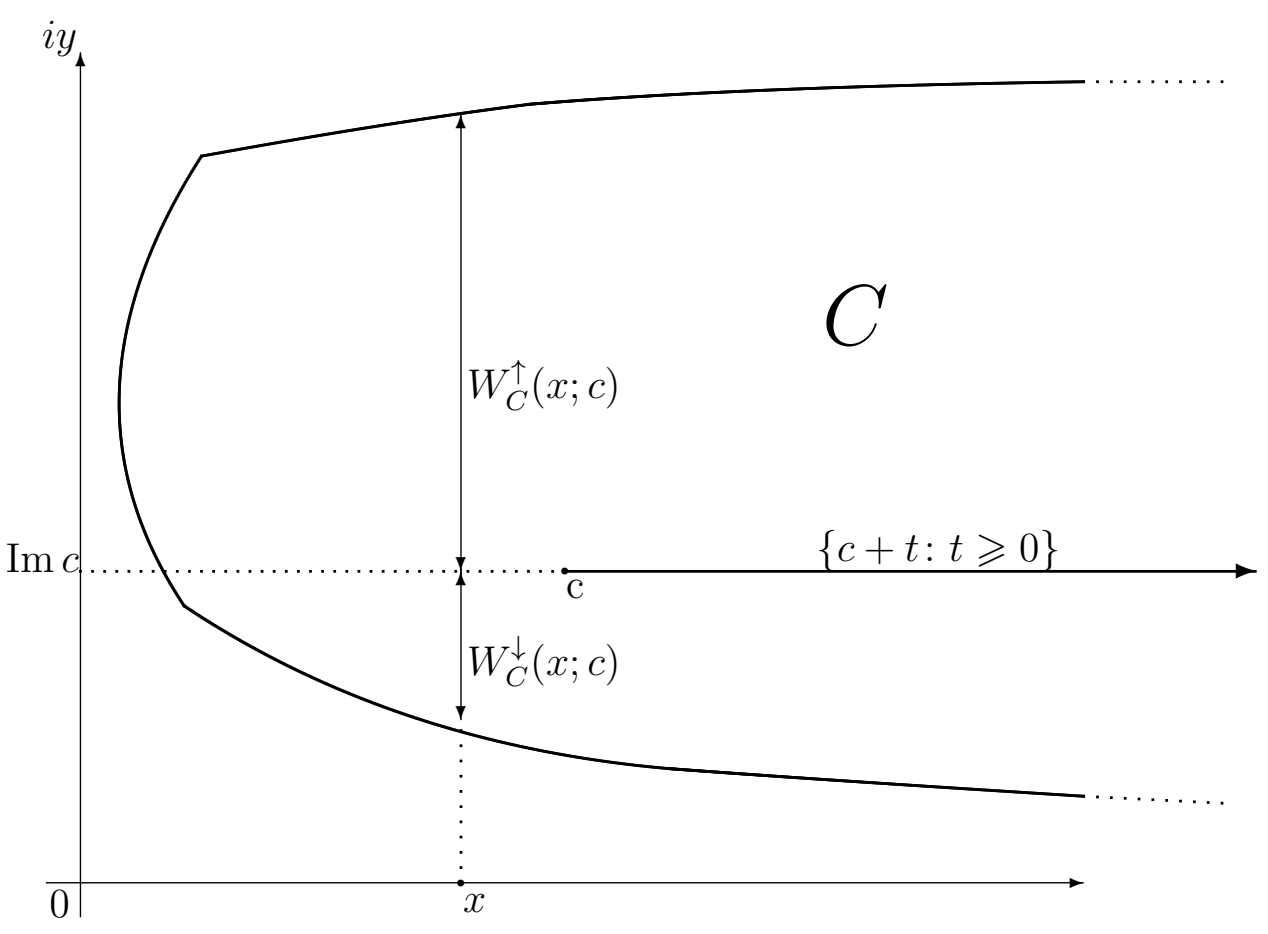

Figure 1. To definition (14) and the proof of Theorem 5, Part (4)

For an unbounded convex set $C \subset \mathbb{C}$ star-shaped in the direction $\theta=0$, the definition of functions $W_{C}^{\uparrow}(\cdot ; c)$ and $W_{C}^{\downarrow}(\cdot ; c)$ w.r.t. a point $c \in C$ in the direction $\theta=0$ is demonstrated on Fig. 1.

Theorem 5. Let $S \subset \mathbb{C}, C$ be a convex set in $\mathbb{C}$.

1. If $C$ has at least two star-shapedness directions $\theta_{1}, \theta_{2} \in \mathbb{R}$ and the difference $\theta_{1}-\theta_{2}$ is not a multiple of $\pi$, and $S$ is bounded, then some shift of set $S$ is contained in $C$.

2. If $0<\theta_{2}-\theta_{1} \leqslant \pi$ and the arc $\smile\left(\theta_{1}, \theta_{2}\right):=\left\{e^{i \theta}: \theta_{1}<\theta<\theta_{2}\right\}$, is contained in $0^{+} C$ and also $\smile\left(\theta_{1}^{\prime}, \theta_{2}^{\prime}\right) \supset 0^{+} S$ and $\theta_{1}<\theta_{1}^{\prime}<\theta_{2}^{\prime}<\theta_{2}$, then some shift of set $S$ is contained in $C$.

3. If set $C$ is closed and has two different star-shapedness directions $\theta_{1}$ and $\theta_{2}$ up to an additive term multiple of $2 \pi$ and the difference $\theta_{2}-\theta_{1}$ is a multiple of $\pi$, but not a multiple of $2 \pi$ (for the sake of definiteness we consider $\theta_{1}=0$ and $\theta_{2}=\pi$ ) then $C$ is a horizontal strip of finite width $b_{C}=B_{C}(\pi / 2)$ and some shift of set $S$ is contained in $C$ if and only if breadth $B_{S}(\pi / 2)$ of set $S$ in the direction $\pi / 2$ does not exceed width $b_{C}$ of strip $C$.

4. If set $C$ is closed and has one star-shapedness direction $\theta=0$ up to an additive term multiple of $2 \pi$, then some shift of set $S$ is contained in $C$ if and only if there exist number $s \in \mathbb{C}, c \in C$ and $x_{0} \in \mathbb{R}$ such that the inequalities

$$
\left\{\begin{array}{lll}
W_{S}^{\uparrow}(x ; s) \leqslant W_{C}^{\uparrow}\left(x+x_{0} ; c\right) & \text { for each } & x \in \mathbb{R}, \\
W_{S}^{\downarrow}(x ; s) \leqslant W_{C}^{\downarrow}\left(x+x_{0} ; c\right) & \text { for each } & x \in \mathbb{R},
\end{array}\right.
$$

hold true.

Proof. 1. By the assumption of first statement of Theorem 5, convex set $C$ contains a non-zero angle in which we can always put bounded set $S$ by a parallel shift.

2. Under the hypothesis of statement 2 we consider numbers $\theta_{1}^{\prime \prime}, \theta_{2}^{\prime \prime}$ satisfying inequalities $\theta_{1}<\theta_{1}^{\prime \prime}<\theta_{1}^{\prime}<\theta_{2}^{\prime}<\theta_{2}^{\prime \prime}<\theta_{2}$. By the definition of star-shapedness direction, it is easy to see that set $C$ contains some shift of angle $\angle\left[\theta_{1}^{\prime \prime}, \theta_{2}^{\prime \prime}\right]:=\left\{r e^{i \theta}: r \geqslant 0, \theta_{1}^{\prime \prime} \leqslant \theta \leqslant \theta_{2}^{\prime \prime}\right\}$, while some shift of set $S$ is contained in the angle $\angle\left[\theta_{1}^{\prime}, \theta_{2}^{\prime}\right] \subset \angle\left[\theta_{1}^{\prime \prime}, \theta_{2}^{\prime \prime}\right]$. The proof of statement 2 is complete.

3. Under the hypothesis of statement 3, for each point $c \in C$ closed convex set $C$ contains straight line $l_{0}(c)$, i.e., the horizontal straight line passing point $c$ [2, Thm. 8.3], [1, Prop. 1]. 
Such property is possessed only the plane, a half-plane with the boundary parallel to the real axis, and horizontal strip of finite width. But the plane and half-plane have more than two star-shapedness directions (up to a number multiple of $2 \pi$ ). Thus, $C$ is indeed a horizontal strip of finite width $b_{C}=B_{C}(\pi / 2)$. Now the concluding part of statement 3 on the shift of set $S$ is obvious.

4. Under the hypothesis of statement 4 (it is useful to bear Fig. 1 in mind), let us prove the sufficiency. At the first step a shift of plane $\mathbb{C}$ together with set $S$ by the number $c-s$ overlap points $s$ and $c$, while set $S$ is shifted into the set $S^{\prime}:=S+(c-s)$, which by (15) satisfies inequalities

$$
\left\{\begin{array}{lll}
W_{S^{\prime}}^{\uparrow}(x ; c) \leqslant W_{C}^{\uparrow}\left(x+x_{0} ; c\right) & \text { for each } & x \in \mathbb{R}, \\
W_{S^{\prime}}^{\downarrow}(x ; c) \leqslant W_{C}^{\downarrow}\left(x+x_{0} ; c\right) & \text { for each } & x \in \mathbb{R} .
\end{array}\right.
$$

for some $x_{0} \in \mathbb{R}$.

We observe that by the convexity of $C$, functions of cut upper and lower width $W_{C}^{\uparrow}(x, s)$ and $W_{C}^{\uparrow}(x, c)$ increase w.r.t. $x$ in the general sense: $\left(x_{1} \leqslant x_{2}\right) \Longrightarrow\left(W_{C}^{\uparrow}\left(x_{1}, c\right) \leqslant W_{C}^{\uparrow}\left(x_{2}, c\right)\right)$ and the same is true for $W_{C}^{\downarrow}(\cdot, c)$. Hence, by shifting set $S^{\prime}=S+(c-s)$ for a sufficiently great number $x_{0}^{\prime} \geqslant x_{0}$, by condition (16) we put the shift $S+(c-s)+x_{0}^{\prime}$ into $C$.

The necessity of (15) for some $s \in \mathbb{C}, c \in C, x_{0} \in \mathbb{R}$ is rather obvious in view of definition (14) for functions of cut upper and lower width. The proof is complete.

Example 1. This example shows that in statement 4 of Theorem 5 cut upper and lower width of sets $S$ and $C$ can not be replaced by the length of cross-sections

$$
W_{S}(x):=\sup \left\{\left|\operatorname{Im} z_{1}-\operatorname{Im} z_{2}\right|: z_{1}, z_{2} \in S, \operatorname{Re} z_{1}=\operatorname{Re} z_{2}=x\right\}
$$

and $W_{C}(x)$ even for a convex set $S$. It is sufficient to consider the sets

$$
\begin{aligned}
& S:=\{x+i y \in \mathbb{C}: x, y \in \mathbb{R}, x \geqslant 0,0 \leqslant y \leqslant \arctan x\}, \\
& C:=\{x+i y \in \mathbb{C}: x, y \in \mathbb{R}, x \geqslant 0,-\arctan x \leqslant y \leqslant 0\},
\end{aligned}
$$

having the unique star-shapedness direction $\theta=0$ (up to a number multiple of $2 \pi$ ) and the width $\pi / 2$. At that, $W_{S}(x)=W_{C}(x)=\arctan x$ as $x \geqslant 0$ and $W_{S}(x)=W_{C}(x) \equiv-\infty$ as $x<0$. But none of shifts of $S$ is contained in $C$.

\section{INCOMPLETENESS OF EXPONENTIAL SYSTEMS AND ENTIRE FUNCTIONS OF EXPONENTIAL TYPE}

In this section we demonstrate a connection between previous results on shifts of sets with incompleteness of exponential systems in spaces of functions.

4.1. General case $\mathbb{C}^{n}, n \geqslant 1$. Let $n \in \mathbb{N}, \mathbb{C}^{n}$ be an $n$-dimensional complex space over field $\mathbb{C}$ equipped by the Euclidean metrics of space $\mathbb{R}^{2 n}$, i.e., $\mathbb{C}^{n}$ is identified with $\mathbb{R}^{2 n}$ : each point

$$
z=\left(z_{1}, \ldots, z_{n}\right) \in \mathbb{C}^{n}, \quad z_{k}=x_{k}+i y_{k}, \quad x_{k}, y_{k} \in \mathbb{R}
$$

is associated with the point $\left(x_{1}, y_{1}, \ldots, x_{n}, y_{n}\right) \in \mathbb{R}^{2 n} ; \bar{z}:=\left(\bar{z}_{1}, \ldots, \bar{z}_{n}\right) \in \mathbb{C}^{n}$. For $\lambda=\left(\lambda_{1}, \ldots, \lambda_{n}\right) \in \mathbb{C}^{n}$ we let

$$
\langle\lambda, z\rangle:=\lambda_{1} z_{1}+\ldots \lambda_{n} z_{n} \in \mathbb{C}, \quad|z|:=\sqrt{\langle z, \bar{z}\rangle}
$$

is the norm in $\mathbb{C}^{n}$. Given an open set $\Omega \subset \mathbb{C}^{n}$, by $\operatorname{Hol}(\Omega)$ we denote the space of holomorphic in $\Omega$ functions equipped with the topology of uniform convergence on compact sets, while for a compact set $C \subset \mathbb{C}^{n}$ by $\operatorname{CHol}(C)$ we denote the Banach space of functions $f: C \rightarrow \mathbb{C}$ continuous on $C$ and holomorphic in the interior int $C$, if it is non-empty, with the standard norm

$$
\|f\|_{\mathrm{CHol}(C)}:=\sup \{|f(z)|: z \in \mathbb{C}\} .
$$


The space of linear continuous functions on $\mathrm{CHol}(C)$ is formed by complex-valued of Radon measures $\mu$ with support supp $\mu \subset C$ [17, Appendix A]. Such measure is not unique for each functional. Moreover, as $n>1$, one can not state that given a linear continuous functional on $\mathrm{CHol}(C)$, there exists a measure with the smallest support (w.r.t. the inclusion) representing this functional [18, Ch. 8]. The characteristic function (Fourier-Borel transform, or FourierLaplace transform, or Laplace transform) of functional-measure $\mu$ is the function

$$
L_{\mu}(\lambda):=\mu\left(e^{\langle\lambda, \cdot\rangle}\right)=\int e^{\langle\lambda, z\rangle} d \mu(z), \quad \lambda \in \mathbb{C}^{n} .
$$

This is an entire function of exponential type, i.e.,

$$
\limsup _{|\lambda| \rightarrow \infty} \frac{\log \left|L_{\mu}(\lambda)\right|}{|\lambda|}<\infty
$$

The class of all entire functions of exponential type is indicated by Ent $[1, \infty)$. If characteristic function $L_{\mu}$ is non-zero, then the functional generated by measure $\mu$ on $\operatorname{CHol}(C)$ is non-zero.

Let $\mathbb{Z}_{+}:=\{0\} \cup \mathbb{N}$. Function-divisor on $\mathbb{C}^{n}$ is the mapping $\Lambda: \mathbb{C}^{n} \rightarrow \mathbb{Z}_{+}$and the support of divisor is indicated as usually by $\operatorname{supp} \Lambda \subset \mathbb{C}^{n}$. Let

$$
p=\left(p_{1}, \ldots, p_{n}\right) \in \mathbb{Z}_{+}^{n}, \quad z=\left(z_{1}, \ldots, z_{n}\right) \in \mathbb{C}^{n}, \quad z^{p}:=\prod_{k=1}^{n} z_{k}^{p_{k}} .
$$

Under these notations each divisor $\Lambda$ on $\mathbb{C}^{n}$ (cf. [16, Ch. 4]) is associated with the system of (multiple) exponentials on $\mathbb{C}^{n}$

$$
\operatorname{Exp}^{\Lambda}:=\left\{z^{p} e^{\langle\lambda, z\rangle}: z \in \mathbb{C}^{n}, \lambda \in \operatorname{supp} \Lambda, p_{1}+\cdots+p_{n} \leqslant \Lambda(\lambda)-1\right\} .
$$

A function $L \in \operatorname{Ent}[1, \infty)$ can be associated with zeroes divisor Zero $_{L}: \mathbb{C}^{n} \rightarrow \mathbb{Z}_{+}$which is equal to the order of zero of function $L$ at $z \in \mathbb{C}^{n}$ for each point $z$. Given an arbitrary divisor $\Lambda$, in what follows we write $\Lambda \leqslant \operatorname{Zero}_{L_{\mu}}$ if $\Lambda(\lambda) \leqslant \operatorname{Zero}_{L_{\mu}}(\lambda)$ for each $\lambda \in \mathbb{C}^{n}$. It is well-known ([16, Thm. 1.1.2]) that if there exists a measure $\mu$ with supp $\mu \subset C$ and non-zero characteristic function $L_{\mu}$ defined by (17) such that $\Lambda(\lambda) \leqslant \operatorname{Zero}_{L_{\mu}}(\lambda)$ for each $\lambda \in \mathbb{C}^{n}$, then system $\operatorname{Exp}^{\Lambda}$ is incomplete in space $\mathrm{CHol}(C){ }^{1}$

A complex-valued measure $\mu$ defined on $\mathbb{C}^{n}$ is concentrated on set $S \subset \mathbb{C}^{n}$ if for each $A \subset \mathbb{C}^{n}$ the identity $\mu(A)=\mu(A \cap S)$ holds true.

Theorem 6. Let $n \in \mathbb{N}, C$ be a convex compact set in $\mathbb{C}^{n}$, and $\mu_{1}, \mu_{2}, \ldots$ be at most countable sequence of complex-valued Radon measures concentrated respectively on sets $S_{1}, S_{2}, \cdots \subset$ $\mathbb{C}^{n}$. If the family $\mathscr{S}=\left\{S_{1}, S_{2}, \ldots\right\}$ satisfies at least on four equivalent statements of Theorem 1, the series $\sum_{k \geqslant 1} \mu_{k}$ *-weakly converges (in the space of continuous functions) to measure $\mu$, and

$$
\sum_{k \geqslant 1} \mu_{k}\left(\mathbb{C}^{n}\right) \neq 0
$$

then under notations (17), for each divisor $\Lambda \leqslant \operatorname{Zero}_{L_{\mu}}$ exponential system $\operatorname{Exp}^{\Lambda}$ is incomplete in space $\mathrm{CHol}(C)$.

Proof. Under *-weak convergence of series $\sum_{k \geqslant 1} \mu_{k}$ to measure $\mu$, the support of measure $\mu$ is contained in the closure of union $S=\bigcup_{k \geqslant 1} S_{k}$ and it is easy to show that in notations (17) the series

$$
\sum_{k \geqslant 1} L_{\mu_{k}}=L_{\mu}
$$

\footnotetext{
${ }^{1} \mathrm{~A}$ system of vectors is incomplete in a topological vector space if the closure of its linear span does not coincide with the space.
} 
converges to function $L_{\mu} \in \operatorname{Ent}[1, \infty)$ uniformly on compact sets in $\mathbb{C}^{n}$ and by $(18)$ we have $L_{\mu}(0) \neq 0$. If a shift $C+a$ of set $C$ covers all $S_{k}$ simultaneously, the same shift $C+a$ covers also cl $S$. Then the non-zero function

$$
e^{\langle-a, \cdot\rangle} \cdot L_{\mu} \in \operatorname{Ent}[1, \infty)
$$

is the characteristic function of measure $\mu_{a}$ defined by the rule $\mu_{a}(A)=\mu(A-a)$, where $A$ is an arbitrary Borel set in $\mathbb{C}^{n}$, and measure $\mu_{a}$ with support supp $\mu_{a} \subset C$ generates a non-zero functional. This functional annihilates the exponential system with the divisor of exponents coinciding with the divisor of zeroes of function (20), which is equal Zero $L_{\mu}$. This functional thus annihilates exponential system $\operatorname{Exp}^{\Lambda}$ since $\Lambda \leqslant \operatorname{Zero}_{L_{\mu}}$. Therefore, system $\operatorname{Exp}^{\Lambda}$ is incomplete in $\mathrm{CHol}(C)$ [16, Thm. 1.1.2].

Remark 1. For arbitrary $\lambda_{0} \in \mathbb{C}^{n}$ condition (18) can be replaced by

$$
\sum_{k \geqslant 1} \int e^{\left\langle\lambda_{0}, z\right\rangle} d \mu_{k}(z) \neq 0 .
$$

We can also formulate a weaker theorem similar to Theorem 6, but this theorem can be formulated only in terms of entire functions of exponential type and their radial regularized growth indicators.

First of all we note that the opposite to (17) is valid: if $L \in \operatorname{Ent}[1, \infty)$, then for $L$ there exists a (non-unique) measure $\mu$ with a compact support in $\mathbb{C}^{n}$ such that $L=L_{\mu}$ in notations (17). For each function $L \in \operatorname{Ent}[1, \infty)$, we introduce an upper semi-continuous function [18, Ch. I, Sect. 8]

$$
h_{r}^{*}(z, L):=\limsup _{z^{\prime} \rightarrow z} \limsup _{t>0, t \rightarrow+\infty} \frac{\log \left|L\left(t z^{\prime}\right)\right|}{t}
$$

called radial regularized growth indicator as entire function $L$ has order 1 . If for a compact set $C \subset \mathbb{C}^{n}$ with the support function $H_{C}$ (we identify $\mathbb{C}^{n}$ with $\mathbb{R}^{2 n}$ ) and function $L \in \operatorname{Ent}[1, \infty$ ) the inequality $h_{r}^{*}(\bar{z}, L) \leqslant H_{C}(z), z \in \mathbb{C}^{n}$, holds true, then by Martino-Erenpreiss-Polya theorem [18, Thm. 8.9], [19, Thm. 12.3], for each domain $\Omega \supset C$, function $L$ is the characteristic function of some measure $\mu$ with compact support supp $\mu \subset \Omega$.

Theorem 7. Let $n \in \mathbb{N}, C$ be a compact set in $\mathbb{C}^{n}$,

$$
L_{1}, L_{2}, \ldots \in \operatorname{Ent}[1, \infty)
$$

be a finite sequence of non-zero function on $\mathbb{C}^{n}$ with radial regularized growth indicators $h_{r}^{*}\left(\cdot, L_{k}\right), k=1,2, \ldots$, and for each $k$ and for some continuous positive homogeneous sublinear function on $\mathbb{R}^{2 n}$ identified with $\mathbb{C}^{n}$, i.e., for support function $H_{S_{k}}$ of some convex closed set $S_{k}$ the inequality $h_{r}^{*}\left(\bar{z}, L_{k}\right) \leqslant H_{S_{k}}(z)$ holds true for each $z \in \mathbb{C}^{n}$. If family $\mathscr{S}=\left\{S_{1}, S_{2}, \ldots\right\}$ satisfies at least one of four equivalent statement of Theorem 1 and

$$
\sum_{k \geqslant 1} L_{k}=L
$$

is a non-zero function, then for each divisor $\Lambda \leqslant \mathrm{Zero}_{L}$, system $\operatorname{Exp}^{\Lambda}$ is incomplete in space $\operatorname{Hol}(\Omega)$ for each domain $\Omega \supset C$.

Proof. Let a shift $C+a$ of compact set $C$ covers all $S_{k}$, and the domain $\Omega+a$ contains $C+a$. Then by Martino-Erenpreiss-Polya theorem, for some measure $\mu$ with a compact support supp $\mu \subset \Omega+a$ in notations $(17) L=L_{\mu}$. Therefore, exponential system Exp ${ }^{\text {Zero }_{L}}$ is annihilates by a non-zero functional-measure $\mu$ on $\operatorname{Hol}(\Omega+a)$. It means that system $\operatorname{Exp}^{\Lambda}$ as $\Lambda \leqslant \operatorname{Zero}_{L}$ is incomplete in space $\operatorname{Hol}(\Omega+a)$. Hence, system $\operatorname{Exp}^{\Lambda}$ is incomplete in $\operatorname{Hol}(\Omega)$. The proof is complete. 
Remark 2. In Theorem 7 , the sequence of functions in (22) can be infinite (countable), but at that one should assume rather strict convergence of the series in (23). For instance, it is sufficient to assume that this series converges uniformly on compact sets in $\mathbb{C}^{n}$ and the estimate

$$
\left|\sum_{1 \leqslant k \leqslant N} L_{k}(z)\right| \leqslant M \exp \left(H_{\mathrm{co}_{k} S_{k}}(\bar{z})\right), \quad z \in \mathbb{C}^{n}, \quad M \text { is a constant. }
$$

holds true uniformly in $N$. We can strengthen Theorem 7 in another direction, namely: system $\operatorname{Exp}^{\Lambda}$ is incomplete in space $\operatorname{Hol}(C)$ of functions holomorphic in the vicinity of compact set $C$ with the natural topology of inductive limit (cf. [16], [18], [19]).

4.2. Planar case $n=1$. As $n=1$, the treatment of some objects appearing in the formulation of Theorems 6 and 7 is slightly simplified.

Instead of the divisor function, it is reasonable to consider at most countable sequence of points $\Lambda=\left\{\lambda_{k}\right\}_{k \geqslant 1} \subset \mathbb{C}$, among which there can be repeating ones, but sequence $\Lambda$ has no limiting points in $\mathbb{C}$. With sequence $\Lambda$, we associate the system of (multiple) exponentials

$$
\operatorname{Exp}^{\Lambda}:=\left\{z^{p} e^{\lambda_{k} z}: z \in \mathbb{C}, 0 \leqslant p \leqslant n_{\Lambda}\left(\lambda_{k}\right)-1\right\},
$$

where $n_{\Lambda}(\lambda)$ is the amount of repetitions of a point $\lambda \in \mathbb{C}$ in sequence $\Lambda$. To a non-zero function $L \in \operatorname{Ent}[1, \infty)$, a sequence of zeroes Zero $_{L}$ counted with orders taken into account. At that, $\Lambda \leqslant \operatorname{Zero}_{L}$ means $n_{\Lambda}(\lambda) \leqslant n_{\text {Zero }_{L}}(\lambda)$ for each $\lambda \in \mathbb{C}$. Under such treatment the phrase "... for each divisor $\Lambda \leqslant \operatorname{Zero}_{L_{\mu}} \ldots$ " in the conclusion of Theorem 6 should be replaced by "...for each sequence $\Lambda \leqslant \operatorname{Zero}_{L_{\mu}} \ldots$.".

Concerning Theorem 7, instead of radial regularized growth indicator as entire function $L \in \operatorname{Ent}[1, \infty)$ has order 1 , we can consider the growth indicator

$$
h(\theta, L):=\limsup _{t>0, t \rightarrow+\infty} \frac{\log \left|L\left(t e^{i \theta}\right)\right|}{t}, \quad \theta \in \mathbb{R},
$$

which is a continuous $2 \pi$-periodic trigonometrically convex function [7], [8], [16], which the support function of some convex compact set (indicator diagram), or is the support function $h_{S}(\theta) \equiv h(-\theta, L)$ of the adjoint diagram $S$ of function $L$. Then Theorem 7 can be reformulated as

Theorem 8. Let $C$ be a convex compact set in $\mathbb{C},(22)$ be a finite sequence of non-zero functions on $\mathbb{C}$ with adjoint diagrams $S_{k}, k=1,2, \ldots$, respectively. If family $\mathscr{S}=\left\{S_{1}, S_{2}, \ldots\right\}$ satisfies at least one of four equivalent statements of Theorem 2 and function $L$ in (23) is a non-zero function, then for each sequence $\Lambda \leqslant Z^{2} \operatorname{ero}_{L}$, system $\operatorname{Exp}^{\Lambda}$ is incomplete in $\operatorname{Hol}(\Omega)$ for each domain $\Omega \supset C$.

Remark 3. Remark 2 is still valid for Theorem 8.

The author is deeply grateful to A.S. Krivosheev for useful discussions of certain issues related with entire functions of many variables.

\section{BIBLIOGRAPHY}

1. B.N. Khabibullin. Helly's Theorem and shifts of sets. I // Ufimskij Matem. Zhurn. 6:3, 98-111 (2014). [Ufa Math. J. 6:3, 95-107 (2014).]

2. R.T. Rockafellar. Convex analysis. Princeton Univ. Press, Princeton (1970).

3. K. Leichtweiß. Konvexe mengen. VEB Deutscher Verlag der Wissenschaften, Berlin (1980). (in German).

4. V.M. Tikhomirov. Convex analysis // Itogi Nauki Tekh. Ser. Sovrem. Probl. Mat. Fundam. Napravleniya. 14, 5-101 (1986). [Encycl. Math. Sci. 14, 1-92 (1990).]

5. G.G. Magaril-Il'yaev, V.M. Tikhomirov. Convex analysis and its applications. Editorial URSS, Moscow (2000). (in Russian). 
6. E.S Polovinkin, M.V. Balashov. Elements of convex and strongly convex analysis. Fizmatlit, Moscow (2004). (in Russian).

7. B.Ya. Levin. Distribution of the zeros of entire functions. Fizmatgiz, Moscow (1956). [Mathematische Lehrbücher und Monographien. II. Abt. Band 14. Akademie-Verlag, Berlin (1962). (in German).]

8. B.Ya. Levin. Lectures on entire functions. Transl. Math. Monographs. V. 150. Amer. Math. Soc. Providence, RI. (1996).

9. S.N. Chernikov. Linear inequalities. Nauka, Moscow (1968). (in Russian).

10. T. Bonnesen, W. Fenchel. Theory of convex bodies. BCS Associates, Moscow, Idaho, USA (1987).

11. H. Hadwiger Vorlesungen über Inhalt, Oberfläche und Isoperimetrie. Springer, Berlin (1957). (in German).

12. L. Santaló. Integral geometry and geometric probability. Addison-Wesley Publishing Company, Reading (1976).

13. B.N. Khabibullin. On the growth of exponential-type entire-functions along the imaginary axis // Dokl. AN SSSR. 302:2, 270-273 (1988). (in Russian).

14. B.N. Khabibullin. On the growth of entire functions of exponential type along the imaginary axis // Matem. Sbornik. 180:5, 706-719 (1989). [Math. USSR-Sbornik 67:1, 149-163 (1990).]

15. B.N. Khabibullin. On the growth of the entire functions of exponential type with given zeros along a line // Anal. Math. 17:3, 239-256 (1991). (in Russian).

16. B.N. Khabibullin. Completeness of exponential systems and uniqueness sets. 4th ed. Bashkir State University, Ufa (2012). (in Russian).

17. T. Ransford. Potential Theory in the Complex Plane. Cambridge Univ. Press, Cambridge (1995).

18. P. Lelong, L. Gruman. Entire functions of several complex variables. Springer, Berlin (1986).

19. V.V. Napalkov. Convolution equations in multi-dimensional spaces. Nauka, Moscow (1982). (in Russian).

Bulat Nurmievich Khabibullin,

Bashkir State University,

Z. Validi str., 32,

450074, Ufa, Russia

E-mail: Khabib-Bulat@mail.ru 